\begin{tabular}{|c|l|}
\hline Title & Solution landscapes of the diblock copolymer-homopolymer model under two-dimensional confinement \\
\hline Author(s) & Xu, Zhen; Han, Y ucen; Y in, Jianyuan; Yu, Bing; Nishiura, Y asumasa; Zhang, Lei \\
\hline Citation & $\begin{array}{l}\text { Physical Review E, 104(1), 014505 } \\
\text { https://doi.org/10.1103/PhysRevE.104.014505 }\end{array}$ \\
\hline Issue Date & 2021-07-29 \\
\hline Doc URL & http://hdl.handle.net/2115/82643 \\
\hline Rights & Copyright 2021 by The A merican Physical Society. \\
\hline Type & article \\
\hline File Information & PhysRevE.104.014505.pdf \\
\hline
\end{tabular}

Instructions for use 


\title{
Solution landscapes of the diblock copolymer-homopolymer model under two-dimensional confinement
}

\author{
Zhen Xu $\odot,{ }^{1}$ Yucen Han, ${ }^{2}$ Jianyuan Yin, ${ }^{3}$ Bing Yu $\bullet,{ }^{3}$ Yasumasa Nishiura, ${ }^{4,}$ and Lei Zhang $\oplus^{1,5, \dagger}$ \\ ${ }^{1}$ Beijing International Center for Mathematical Research, Peking University, Beijing 100871, China \\ ${ }^{2}$ Department of Mathematics and Statistics, University of Strathclyde, Glasgow G1 1XQ, United Kingdom \\ ${ }^{3}$ School of Mathematical Sciences, Peking University, Beijing 100871, China \\ ${ }^{4}$ Research Center of Mathematics for Social Creativity, Research Institute for Electronic Science, \\ Hokkaido University, N12W7, Kita-Ward, Mid-Campus Open Laboratory Building No.2, Sapporo 060-0812, Japan \\ ${ }^{5}$ Center for Quantitative Biology, Peking University, Beijing 100871, China
}

(Received 30 March 2021; accepted 8 July 2021; published 29 July 2021)

\begin{abstract}
We investigate the solution landscapes of the confined diblock copolymer and homopolymer in twodimensional domain by using the extended Ohta-Kawasaki model. The projection saddle dynamics method is developed to compute the saddle points with mass conservation and construct the solution landscape by coupling with downward and upward search algorithms. A variety of stationary solutions are identified and classified in the solution landscape, including Flower class, Mosaic class, Core-shell class, and Tai-chi class. The relationships between different stable states are shown by either transition pathways connected by index-1 saddle points or dynamical pathways connected by a high-index saddle point. The solution landscapes also demonstrate the symmetry-breaking phenomena, in which more solutions with high symmetry are found when the domain size increases.
\end{abstract}

DOI: 10.1103/PhysRevE.104.014505

\section{INTRODUCTION}

The diblock copolymers are composed of two different blocks linked together through covalent bonding. A large number of copolymers interact with each other to produce a wide variety of microstructures, resulting from a compromise between phase segregation and polymer architecture [1-4]. The diblock copolymer materials have aroused great interest in both industrial and theoretical research. Moreover, an important commercialized application of copolymers is thermoplastic elastomers, which have been widely used as jelly candles, outer coverings for optical fiber cables, adhesives, bitumen modifiers, etc. [5,6]. Much scientific interest on the self-assembly of block copolymers is due to the pattern formation and the potential applications of the microstructures led by the confinement mechanism, which restricts degrees of freedom in space and breaks symmetry of the structure [7].

Extensive experimental and theoretical studies have demonstrated that confinement can be used to control the self-assembly of diblock copolymers [8]. The diblock copolymers under different confinements have been well studied, such as two-dimensional (2D) cylindrical confinement [9-12], three-dimensional (3D) cylindrical confinement [13-15], and 3D spherical or polyhedral confinement [16-18]. Many morphologies have been discovered $[8,11,19,20]$, for example, the onion-like and layered structures for symmetric copolymers under spherical confinement in experiments $[21,22]$. These

\footnotetext{
*yasumasa@pp.iij4u.or.jp

†zhangl@math.pku.edu.cn
}

resulting morphologies are often distinctly different from those in the bulk phase [23] and thus defined as the "frustrated phases" [24]. Meanwhile, many mathematical models and numerical studies have been carried out to investigate the confinement of copolymers and their self-assembly, including Monte Carlo simulation [9,14,16,25], cell dynamics simulation [26-29], the self-consistent field theory [30-32], and the phase-field method [33-36].

The first qualitative model for the block copolymers was proposed by Ohta and Kawasaki [37] in the form of a generalized Landau free-energy functional with nonlocal term to describe the linkage between the different blocks in copolymers. In contrast to Leibler's work [38], which proposed a weak-segregation theory, the Ohta-Kawasaki model referred to a strong segregation for the density functional theory. In the strong-segregation limit, the interfacial thickness is sufficiently small compared to the structure-domain dimension. After that, the density functional was carefully rederived in a more mathematical formulation by Nishiura et al. [39], considering a fractional power of the Laplace operator to represent the long range interaction. Compared to the Green functions, this elegant fractional operator is more suitable for variational problems $[21,39]$. With this introduction to mathematical community, some other people then started to work in this direction. For example, Teramoto and Nishiura used this model to study the diblock copolymer problem in a three-dimensional space for the topological properties of the energy minimizers and the morphology transition between them [23]. Choksi and Ren also rederived the density functional by using the self-consistent mean-field theory for microphase of diblock copolymers and blends $[34,40]$. The 
above-mentioned Ohta-Kawasaki model was extended to a system composed of block copolymers and homopolymers with two phase variables to describe the macrophase separation between the copolymers and homopolymers, as well as the microphase separation between the two components of the diblock copolymers $[33,41]$. Hereafter, we call the extended Ohta-Kawasaki free-energy functional as the diblock copolymer-homopolymer (DCH) free-energy functional for simplicity. Multiple stable self-assembled phases can be found by numerically solving two coupled Cahn-Hilliard equations of the DCH free-energy functional, including layers, tennis balls, onions, and multipods under the nano-confinements [16-18,21]. However, there are two remaining questions which need to be addressed. The first question is what are the relationships between different stable states? To answer it, one needs to compute the transition pathways connecting these stable states. The second question is how to efficiently search possible stationary solutions of the DCH free-energy functional? The traditional numerical algorithms need to carefully choose suitable initial conditions, which often requires good intuition of physical systems or the guide from experiments, to find more solutions. It is eager to develop a systematic numerical approach to compute all stationary solutions without tuning unwanted initial guesses.

The stationary solutions correspond to the solutions of the Euler-Lagrange equation of the DCH free-energy functional with the mass conservation. The Euler-Lagrange equation usually has multiple solutions, including both stable/metastable solutions, i.e., local minima, and unstable saddle points of the system. The properties of stationary solutions can be characterized by the Morse index of the solution. The Morse index of a stationary solution is equal to the number of negative eigenvalues of its Hessian matrix [42]. In particular, a local minimum or stable state can be regarded as an index- 0 solution with no unstable directions. Compared to computing a stable state by gradient dynamics, the saddle point is much more difficult to find due to its unstable nature, while often plays critical roles in determining the properties of the model system. For instance, to find the transition pathways between two stable states, one needs to compute the transition state, which is an index- 1 saddle point and the corresponding Hessian matrix has one and only one negative eigenvalue. It has attracted substantial attentions to find multiple stationary solutions of the nonlinear problems [43]. Considerable efforts have been made to develop various numerical algorithms, such as the minimax method [44], the deflation technique [45], the eigenvector-following method [46], and the homotopy method $[47,48]$. In particular, Yin et al. proposed a saddle dynamics (SD) and implemented a high-index optimization-based shrinking dimer method to compute any-index saddle points $[49,50]$. By combining the SD with the downward and upward search algorithms, Yin et al. further constructed a solution landscape, which is a pathway map consisting of all stationary solutions and their connections, for the unconstrained systems [51,52]. It shows a hierarchy structure that starts with a parent state (the highestindex saddle point) and then relates the lower-index saddle points down to the minima. This numerical approach has been successfully applied to the Landau-type free-energy functional, including the defect landscape of confined nematic liquid crystal on a square using a Landau-de Gennes model $[51,53]$ and the transition pathways between period crystals and quasicrystals by applying the Lifshitz-Petrich model [54].

In this paper, we apply the DCH model to investigate the solution landscape of the diblock copolymers and homopolymers in $2 \mathrm{D}$ confinement. To deal with the mass-conservation constraint, instead of using the Cahn-Hilliard dynamics in the $H^{-1}$ inner product for the saddle point calculation, we introduce the projection operator to the SD method in the $L^{2}$ inner product, which only requires a simple linear projection step and avoids the numerical challenge due to the $H^{-1}$ metric. Here, the space $H^{-1}$ is denoted by the dual space to $H_{0}^{1}$.

Applying the PSD method, we systematically construct the solution landscapes with two critical parameters: one represents the preference intensity and the other corresponds to the domain size. A variety of stationary solutions are found in the solution landscapes, including Flower class, Mosaic class, Core-shell class, and Tai-chi class. Furthermore, the solution landscapes demonstrate the relationships between different stable states by either transition pathways connected by index- 1 saddle points or dynamical pathways connected by a high-index saddle point. The solution landscapes also reveal the symmetry-breaking phenomena, in which more solutions with high symmetry are identified when the domain size increases.

The rest of the paper is organized as follows. The DCH model is briefly introduced in Sec. II. The PSD method and the numerical algorithm of construction of a solution landscape are presented in Sec. III. We numerically construct the solution landscapes with different preferences and domain sizes in Sec. IV. Final conclusions and discussions are presented in Sec. V.

\section{DIBLOCK COPOLYMER-HOMOPOLYMER MODEL}

We consider the mixture of $\mathrm{AB}$ diblock copolymers and $\mathrm{C}$ homopolymers [33], with two independent and conserved phase-field order parameters $\eta$ and $\phi . \eta$ represents the macrophase separation with a phase boundary that can be understood as a confining surface, which arises naturally to separate the homopolymer phase from the copolymer phase. The copolymers are assumed to be immersed in an external-medium homopolymers or solvent, such as water. In the copolymer-rich domain, another variable $\phi$ describes the microphase separation between the block $\mathrm{A}$ and block B. When the above two systems interact with one another, the morphologies consist of the confinement surface and copolymer components within the surface, and then undergo a macrophase and microphase separation described by $\eta$ and $\phi$, respectively.

The DCH free-energy functional can be written as a sum of short-range contribution and long-range contribution

$$
F\{\eta ; \phi\}=F_{S}\{\eta ; \phi\}+F_{L}\{\eta ; \phi\}
$$

The short-range contribution $F_{S}$ is given by

$$
F_{S}\{\eta ; \phi\}=\int_{\Omega}\left[\frac{D_{1}}{2}|\nabla \eta|^{2}+\frac{D_{2}}{2}|\nabla \phi|^{2}+W(\eta, \phi)\right] d \mathbf{r}
$$


where $\Omega$ is a Lipschitz-boundary domain in $\mathbb{R}^{2} . D_{1}, D_{2}$ are parameters controlling the size of the macrophase and microphase separation interface, respectively.

The potential is taken as the polynomial form,

$$
\begin{aligned}
W(\eta, \phi)= & \frac{\left(\eta^{2}-1\right)^{2}}{4}+\frac{\left(\phi^{2}-1\right)^{2}}{4} \\
& +b_{1} \eta \phi-\frac{b_{2}}{2} \eta \phi^{2}-\frac{b_{3}}{2} \eta^{2} \phi+\frac{b_{4}}{2} \eta^{2} \phi^{2} .
\end{aligned}
$$

The first two terms in Eq. (3) exhibit double-well potential for $\eta$ and $\phi$, respectively, and the rest terms describe the coupling between the $\mathrm{AB}$ copolymers and the solvent $\mathrm{C}[33,41]$. The coefficients $b_{1}, b_{2}, b_{3}$ and $b_{4}$ are positive constants, which are related to the molecular parameters and could be derived in principle by the generalized method $[33,37,41,55]$. These parameters are chosen so that $W(\eta, \phi)$ has a triplewell structure with three distinct minima corresponding to the phases of block A, block $\mathrm{B}$, and solvent $\mathrm{C}$. Thus, we set $b_{3}=b_{4}=0$ and only change $b_{1}, b_{2}$. When $b_{1}=0, b_{2}=1$, $W(\eta, \phi)$ has three minima at $(-1,0),(1,1)$, and $(1,-1)$, and the free-energy functional is symmetrical corresponding to $\phi$, indicating that $\eta$ or the confining surface has equal preference for positive or negative $\phi$, such as the morphology of layer $[21,22,55]$. Conversely, the nonzero $b_{1}$ would cause symmetry breaking between microphase separated domains, i.e., the selective preference between block A $(\phi>0)$ and block B $(\phi<0)$.

The long-range contribution $F_{L}$ is given by

$$
F_{L}\{\eta ; \phi\}=\int_{\Omega} \frac{\alpha}{2}\left|(-\Delta)^{-\frac{1}{2}}(\phi-\bar{\phi})\right|^{2} d \mathbf{r},
$$

where $\bar{\phi}$ represents the spatial average of $\phi$. In the original paper, the Green functions are used to represent long-range interactions [37], which was replaced with a nonlocal operator, the fractional power of the Laplace operator for variational problems [39]. The long-range contribution prevents the copolymers from forming a large macroscopic domain and brings about many fine structures, such as layers or onions. $\alpha$ is inversely proportional to the square of total chain length of the copolymer and related to the bonding between block $\mathrm{A}$ and block B in copolymers, hence it is a measure of the connectivity between two blocks [56]. When $\alpha=0$, there is no linkage between A and B blocks, and the absence of the nonlocal term will induce the separation macroscopically. If $\alpha \neq 0$, then we have microphases within the copolymer-rich domain and multiple morphologies emerge.

Now we nondimensionalize the system with $\tilde{r}=r / \lambda$, then the rescaled free-energy functional is

$$
\begin{aligned}
\tilde{F}\{\tilde{\eta} ; \tilde{\phi}\}= & \int_{\Omega} \frac{D_{1}}{2}|\tilde{\nabla} \tilde{\eta}|^{2}+\frac{D_{2}}{2}|\tilde{\nabla} \tilde{\phi}|^{2} \\
& +\lambda^{2}\left(\frac{1}{4}\left(\tilde{\eta}^{2}-1\right)^{2}+\frac{1}{4}\left(\tilde{\phi}^{2}-1\right)^{2}\right. \\
& \left.+b_{1} \tilde{\eta} \tilde{\phi}-\frac{1}{2} b_{2} \tilde{\eta} \tilde{\phi}^{2}\right) \\
& +\frac{\alpha \lambda^{4}}{2}\left|(-\tilde{\Delta})^{-\frac{1}{2}}(\tilde{\phi}-\bar{\phi})\right|^{2} d \tilde{r}
\end{aligned}
$$

where $\tilde{\Omega}$ is a unit square $[0,1] \times[0,1]$, and $\lambda$ is the length of the square domain. In what follows, we drop the tildes and all statements are in terms of the rescaled variables. Here both $\eta$ and $\phi$ are the conserved order parameters satisfying

$$
\int_{\Omega}(\eta-\bar{\eta}) d \mathbf{r}=0, \int_{\Omega}(\phi-\bar{\phi}) d \mathbf{r}=0 .
$$

The stationary solutions of the DCH functional with mass conservation are the solutions of the Euler-Lagrange equations as follows:

$$
\begin{aligned}
& \frac{\delta F(\eta, \phi)}{\delta \eta}-\xi_{\eta}=0, \\
& \frac{\delta F(\eta, \phi)}{\delta \phi}-\xi_{\phi}=0 .
\end{aligned}
$$

$\xi_{\eta}$ and $\xi_{\phi}$ are the Lagrangian multipliers to keep the mass conservation.

\section{NUMERICAL METHOD}

\section{A. Projection saddle dynamics method}

To find the multiple stationary solutions of the EulerLagrange Eq. (8) with the mass conservation Eq. (6), we need to develop an efficient numerical algorithm to compute the saddle points with mass conservation. The original SD method is designed for unconstrained gradient systems [50]. Recently, Huang et al. proposed a constrained high-index saddle dynamics method to compute the constrained saddle points and construct the solution landscape with equality constraints by using Riemannian gradient and Hessian [57]. In the DCH model, since the mass conservation is only a linear constraint, we propose a simple PSD method to compute index- $k$ saddle points ( $k$-saddles) with the mass-conservation constraint. Here, the projection is defined as follows:

$$
P(\xi)=\xi-\int_{\Omega} \xi d \mathbf{r}, \xi \in L^{2}(\Omega) .
$$

Both gradient and Hessian of $F(\eta ; \phi)$ are updated by the projected forms. In addition, to eliminate the unphysical directions, we translate the order parameters $\eta$ and $\phi$ to $\hat{\eta}=\eta-\bar{\eta}$ and $\hat{\phi}=\phi-\bar{\phi}$ so that $\int_{\Omega} \hat{\eta} d \mathbf{r}=0, \int_{\Omega} \hat{\phi} d \mathbf{r}=0$. In the following, we also drop the hats and all statements are in terms of the translated variables.

The PSD for computing a mass-conserved $k$-saddle $(k$ PSD) is governed by the following dynamic equations:

$$
\begin{aligned}
\dot{\eta}= & -P \frac{\delta F(\eta ; \phi)}{\delta \eta} \\
& +2 \sum_{j=1}^{k}\left\langle P \frac{\delta F(\eta ; \phi)}{\delta \eta} v_{j}+P \frac{\delta F(\eta ; \phi)}{\delta \phi} w_{j}, v_{j}\right\rangle, \\
\dot{\phi}= & -P \frac{\delta F(\eta ; \phi)}{\delta \phi} \\
& +2 \sum_{j=1}^{k}\left\langle P \frac{\delta F(\eta ; \phi)}{\delta \eta} v_{j}+P \frac{\delta F(\eta ; \phi)}{\delta \phi} w_{j}, w_{j}\right\rangle .
\end{aligned}
$$

Equations (9) allow $[\eta ; \phi]$ to move along an ascent direction on the subspace $\mathcal{V}=\operatorname{span}\left(\left[v_{1} ; w_{1}\right], \cdots,\left[v_{k} ; w_{k}\right]\right)$, and a 
descent direction on the subspace $\mathcal{V}^{\perp}$, the orthogonal complement space of $\mathcal{V} .\left[v_{i} ; w_{i}\right](i=1 \cdots k)$ are the orthonormal and unit eigenvectors, i.e., $\left\langle\left[v_{i} ; w_{i}\right],\left[v_{j} ; w_{j}\right]\right\rangle=\delta_{i, j}$, corresponding to the smallest $k$ eigenvalues $\lambda_{1}, \cdots, \lambda_{k}$ of the Hessian $\nabla^{2} F(\eta ; \phi)$. They can be obtained via the constrained optimization problem and governed by the following equations:

$$
\begin{aligned}
{\left[\begin{array}{c}
\dot{v}_{i} \\
\dot{w}_{i}
\end{array}\right]=- } & \left(I-\left[\begin{array}{c}
v_{i} \\
w_{i}
\end{array}\right]\left[\begin{array}{c}
v_{i} \\
w_{i}
\end{array}\right]^{T}+2 \sum_{j=1}^{i-1}\left[\begin{array}{c}
v_{j} \\
w_{j}
\end{array}\right]\left[\begin{array}{c}
v_{j} \\
w_{j}
\end{array}\right]^{T}\right) \\
& \left(\begin{array}{c}
P \\
P
\end{array}\right) \nabla^{2} F(\eta ; \phi)\left[\begin{array}{c}
v_{i} \\
w_{i}
\end{array}\right], i=1, \cdots, k .
\end{aligned}
$$

Here $I$ is the identity operator. The $k$-saddle $[\eta ; \phi]$ and $k$ direction variables $\left[v_{i} ; w_{i}\right]$ are coupled. We note that $v_{i}$ and $w_{i}$ are also needed to apply the projection $P$ to keep mass conservation, namely $\int_{\Omega} v_{i} d \mathbf{r}=\int_{\Omega} w_{i} d \mathbf{r}=0$.

To avoid the direct calculation of Hessians, we approximate Hessian by using the central difference scheme for directional derivations on $k$ dimers centered at $[\eta ; \phi][49,50]$. The $i$ th dimer has a direction of $\left[v_{i} ; w_{i}\right]$ with a small dimer length $2 l$ and then $\nabla^{2} F(\eta ; \phi)$ is approximated by

$$
\begin{aligned}
& \nabla^{2} F(\eta ; \phi)\left[\begin{array}{ll}
v_{i} & w_{i}
\end{array}\right] \\
& \quad \approx \frac{\nabla F\left(\left[\begin{array}{l}
\eta+l v_{i} \\
\phi+l w_{i}
\end{array}\right]\right)-\nabla F\left(\left[\begin{array}{c}
\eta-l v_{i} \\
\phi-l w_{i}
\end{array}\right]\right)}{2 l} .
\end{aligned}
$$

We choose periodic boundary conditions and apply the Fourier spectral method for the space discretization on $\Omega$. The numerical simulations are performed on a 2D $128 \times 128$ grid which was verified to give well-resolved numerical results. We use the explicit Euler scheme and Barzilai-Borwein gradient method to determine the step sizes for time discretization of Eq. (9) [58]. Furthermore, we apply the locally optimal block preconditioned conjugate gradient (LOBPCG) method [59] to compute the smallest $k$ eigenvalues and the corresponding eigenvectors of the Hessian.

The initial condition is given as

$[\eta(0) \phi(0)]=\left[\begin{array}{ll}\eta^{0} & \phi^{0}\end{array}\right],\left[v_{i}(0) w_{i}(0)\right]=\left[\begin{array}{ll}v_{i}^{0} & w_{i}^{0}\end{array}\right], i=1, \cdots, k$,

where $\left[\eta^{0} ; \phi^{0}\right]$ are zero mean, and $\left[v_{1}^{0} ; w_{1}^{0}\right], \cdots,\left[v_{k}^{0} ; w_{k}^{0}\right]$ are the unit orthogonal vectors with zero mean.

\section{B. Algorithm for the solution landscape}

The solution landscape of the DCH model is constructed via two algorithms: downward search and upward search [51]. The downward search algorithm enables us to efficiently search for all connected low-index saddles and minima from a high-index saddle, and the upward search algorithm aims to find the possible higher-index saddles. The details of two algorithms coupled with PSD method are as follows:

\section{Downward search algorithm}

Assuming we have an $m$-saddle $\left[\eta^{\star} ; \phi^{\star}\right]$ and the $m$ normalized vectors $\left[v_{1}^{\star} ; w_{1}^{\star}\right], \cdots,\left[v_{m}^{\star} ; w_{m}^{\star}\right]$ corresponding to the $m$ negative eigenvalues of the Hessian matrix $\nabla^{2} F\left(\eta^{\star} ; \phi^{\star}\right)$. We then apply the $(m-1)$-PSD Eq. (9) to search the $(m-1)$ saddles by choosing $\left[\eta^{\star} ; \phi^{\star}\right] \pm \epsilon\left[v_{m}^{\star} ; w_{m}^{\star}\right]$ as an initial state and $\left[v_{i}^{\star} ; w_{i}^{\star}\right], \cdots,\left[v_{m-1}^{\star} ; w_{m-1}^{\star}\right]$ as initial unstable directions.
Once a $(m-1)$-saddle is obtained, we continue to apply the $(m-2)$-PSD to search the $(m-2)$-saddles.

By repeating the above procedure, we can establish a systematic search for all saddle points branched from this $m$-saddle as a parent and to construct a family tree that eventually connects to the local minima.

\section{Upward search algorithm}

If the parent state (the highest-index saddle point) is unavailable beforehand or multiple parent states exist, then one can conduct the upward search to find the high-index saddle points starting from a local minimum or a low-index saddle point.

Starting from an $m$-saddle $\left[\eta^{\star} ; \phi^{\star}\right]$, we apply the $(m+1)$ PSD to search an $(m+1)$-saddle. The initial state is chosen as $\left[\eta^{\star} ; \phi^{\star}\right] \pm \epsilon\left[v_{m+1}^{\star} ; w_{m+1}^{\star}\right]$, and $\left[v_{1}^{\star} ; w_{1}^{\star}\right], \cdots,\left[v_{m+1}^{\star} ; w_{m+1}^{\star}\right]$ are taken as the initial ascent directions, in which $\left[v_{m+1}^{\star} ; w_{m+1}^{\star}\right]$ is the eigenvector corresponding to the smallest positive eigenvalue of its Hessian matrix.

Each downward search represents the relaxation of a pseudodynamics, the so-called dynamical pathway, starting from a high-index saddle point to a local minimum. By combining the downward search and upward search, we are able to systematically find possible stationary solutions and uncover the connectivity of the solution landscape.

\section{RESULTS}

Now we present the numerical results for the solution landscapes of the diblock copolymers and homopolymers under $2 \mathrm{D}$ confinement. To see the effect of the preference intensity $b_{1}$ and the domain size $\lambda$, we choose three cases: the equal preference, the selective preference, and the equal preference in a larger domain.

\section{A. Solution landscape with equal preference}

In the case of equal preference $\left(b_{1}=0, \lambda=1\right)$, we plot three stable states (Layer $3 Y$, Layer 3B, and Layer 2) in Fig. 1. Some parameters are set as $D_{1}=D_{2}=0.0025, b_{2}=1, \alpha=$ $60, \bar{\eta}=-0.5, \bar{\phi}=0$. Since the preference for block A (yellow) and block B (blue) are equal, Layer $3 Y$ and Layer $3 B$ are a pair of solutions only with block A and B switched, and the two blocks in Layer 2 have the same area and shape. From an energy point of view, Layer 2 is the stable phase with the lowest energy, while Layer $3 Y$ and Layer $3 B$ are the metastable phases.

The solution landscape with equal preference is shown in Fig. 2. The homogeneous phase $(\eta \equiv \bar{\eta}, \phi \equiv \bar{\phi})$ is clearly a trivial solution, which is a 8 -saddle. Using it as the parent state, we are able to find three distinct 5-saddles via the downward search, specifically, Flower 6 looks like a blooming flower with three yellow petals alternating with three blue petals, and Circle $2 Y S$ and Circle $2 B S$ are a pair of solutions due to the equal preference of blue blocks and yellow blocks.

The periodic boundary condition implies that the Hessian at a nonhomogeneous state has at least two zero eigenvalues in most cases, which explains why no 6-saddles or 7-saddle are found in Fig. 2. Down from these 5-saddles, a variety of complex morphologies in Triangle class and Flower 


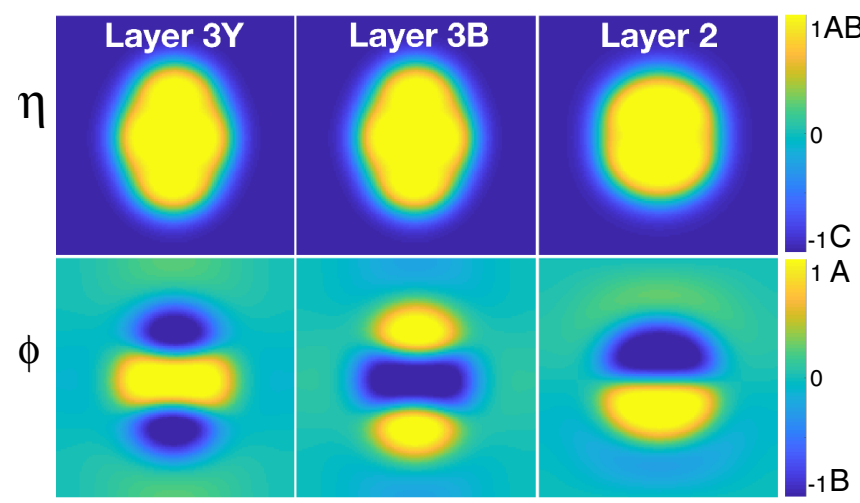

FIG. 1. Three stable states: Layer $3 Y$, Layer $3 B$, and Layer 2 with equal preference. The first row and the second row show the spatial distributions of $\eta$ and $\phi$, respectively. In the plot of $\eta$, and $\mathrm{AB}$ copolymers and $\mathrm{C}$ homopolymers are represented by yellow and blue, respectively. In the plot of $\phi$, block A and block B of copolymers are distinguished by yellow and blue, respectively. All subsequent figures share the same color bar for $\phi$.

class are obtained. In the Triangle class, the shapes of inner blocks are isosceles triangles with the apex angle less than 60 degrees (Triangle $Y 1$ and Triangle B1), isosceles triangles with the apex angle greater than 60 degrees (Triangle $Y 2$ and Triangle B2), and equilateral triangles (Triangle $Y 3$ and Triangle B3). In Flower class, the asymmetric and symmetric flower solutions, Flower $4 N 1$, Flower $4 Y 2$, Flower $4 B 2$, and Flower 4, have two yellow petals alternating with two blue petals. Flower $3 Y$ has one yellow petal between two blue petals and Flower $3 B$ has one blue petal between two yellow petals. The asymmetric Layer $3 N Y$ (Layer $3 N B$ ) is the transition state between the metastable solution Layer $3 Y$ (Layer $3 B$ ) and the stable solution Layer 2 in Fig. 1.

From the solution landscape, we can also extract the transition pathways between three stable states Layer $3 Y$, Layer $3 B$, and Layer 2 (Fig. 3). There are two transition pathways between Layer $3 B$ and Layer 2: Layer $3 B \rightarrow$ Flower $3 B \rightarrow$ Layer 2 and Layer $3 B \rightarrow$ Layer $3 N B \rightarrow$ Layer 2 in Fig. 3(a). The latter one has the smaller energy barrier $\Delta F$ (the energy difference between the transition state and the initial stable state), thus has higher possibility to take place. With the equal preference, it is easy to see the transition pathways between Layer $3 Y$ and Layer 2 are analogous: Layer $3 Y \rightarrow$ Flower $3 Y \rightarrow$ Layer $2(\Delta F=4.1 e-3)$ and Layer $3 Y \rightarrow$ Layer $3 N Y \rightarrow$ Layer $2(\Delta F=3 e-4)$. Figure 3(b) shows the switching process between Layer $3 B$ and Layer $3 Y$, along which blue block and yellow block swap, connected by the transition state Flower 4 with two opposed blue petals and two opposed yellow petals. In the process from Layer $3 B$ to Flower 4, the yellow block on both sides of the blue block push into the middle of blue block. The shape of blue block changes from an ellipse to an hourglass, and is further cut into two small petals. In the process from Flower 4 to Layer $3 Y$, the two yellow petals connect together resulting in a yellow hourglass and relax to an ellipse between two small blue ellipses. From Fig. 2, Layer $3 B$ and Layer $3 Y$ can also be connected via Layer 2, that is, Layer $3 B \rightarrow$ Layer $3 N B \rightarrow$ Layer 2 [Fig. 3(a)] and Layer $2 \rightarrow$ Layer $3 N Y \rightarrow$ Layer $3 Y$.

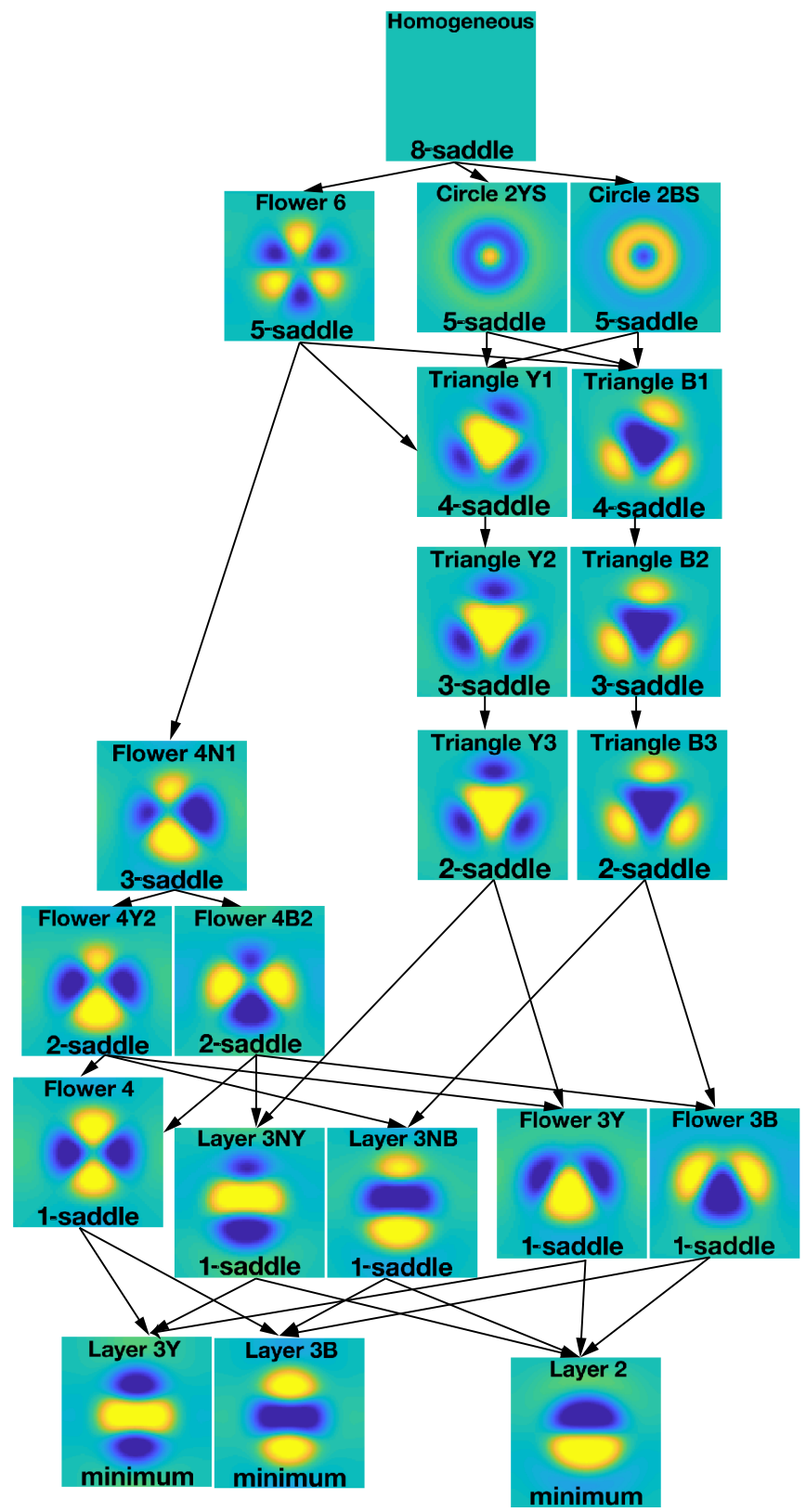

FIG. 2. Solution landscape with equal preference. The height of a phase approximately corresponds to its energy. The $\phi$ plot of each phase is shown in square domain with name on the top and index of corresponding saddle point at the bottom.

In fact, this transition pathway has a lower energy barrier and is more probable than the one in Fig. 3(b).

\section{B. Solution landscape with selective preference}

We next study the solution landscape with selective preference $\left(b_{1}=0.1, \lambda=1\right)$, namely the affinity of the blue block for homopolymers (solvent) is higher than that of the yellow one. This leads to the symmetry breaking of the morphologies in contrast to the one with equal preference. In Fig. 4, the homogeneous phase (8-saddle) is still the parent state, and the transition states between Layer $3 Y$ and Layer $2 Y$ are also Layer $3 N Y$ and Flower $3 Y$. While due to the symmetry 

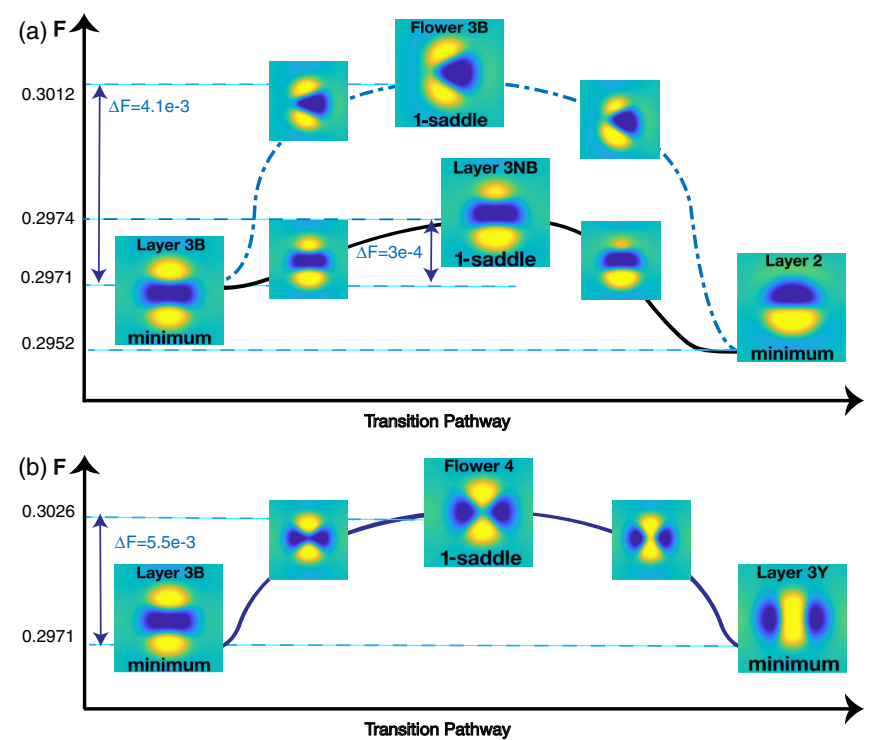

FIG. 3. Transition pathways between stable states with equal preference. (a) Transition pathways between Layer $3 B$ and Layer 2 with the transition state Flower $3 B$ (energy barrier $4.1 e-3$ ) or Layer $3 N B$ (energy barrier $3 e-4$ ). (b) Transition pathway between Layer $3 B$ and Layer $3 Y$ with the transition state Flower 4 (energy barrier $5.5 e-3)$.

breaking, the structure of the solution landscape has changed dramatically. On the one hand, we lose nearly half of the stationary solutions observed in Fig. 2. For instance, F lower 6 in Fig. 2 with alternative yellow and blue blocks merges into Circle $2 Y S$ with blue ring surrounding the yellow blocks at the center. Although Flower 6 disappears in the case of selective preference, Flower 4 still survives with larger blue petals and smaller yellow petals. Moreover, since the area of inner yellow blocks becomes smaller, Flower $4 N 1$ in Fig. 2 merges into Flower $4 Y 2$, and Triangle $Y 1$ and Triangle $Y 2$ in Fig. 2 merge into the regular triangle Triangle $Y 3$. The Layer $3 B$ solution loses stability and becomes an 1-saddle. However, alternative solutions appear. For example, the 5saddle Circle $2 Y S$ connects to a 4-saddle Circle $2 Y B$, and the 1 -saddle $F$ lower $3 Y$ bifurcates into an asymmetric 2-saddle Flower $3 N Y$.

\section{Solution landscape with equal preference in a larger domain}

We further investigate the solution landscape with equal preference in a larger domain $\left(b_{1}=0, \lambda=1.4\right)$. In Fig. 5, the homogeneous state becomes a 12-saddle and more stationary solutions emerge in the solution landscape, such as 9-saddle Pentagon $Y$ with the same symmetric property as a pentagon and 7-saddle Flower 8 with four blue petals and four yellow petals. It is worth mentioning that we find stable solutions such as Dendritic 3B, Layer 4, and Circle 2B. The Dendritic $3 B$ solution, which looks like a steering wheel, appears in the larger domain. This phase is a 2D analogy to the multipod phase in 3D [21]. The layer numbers of the Layer-class solutions also increase to 3 (e.g., Layer $3 B$ ) and 4 (e.g., Layer 4 ). The Circle $2 B$ becomes a local minimum in the larger domain.

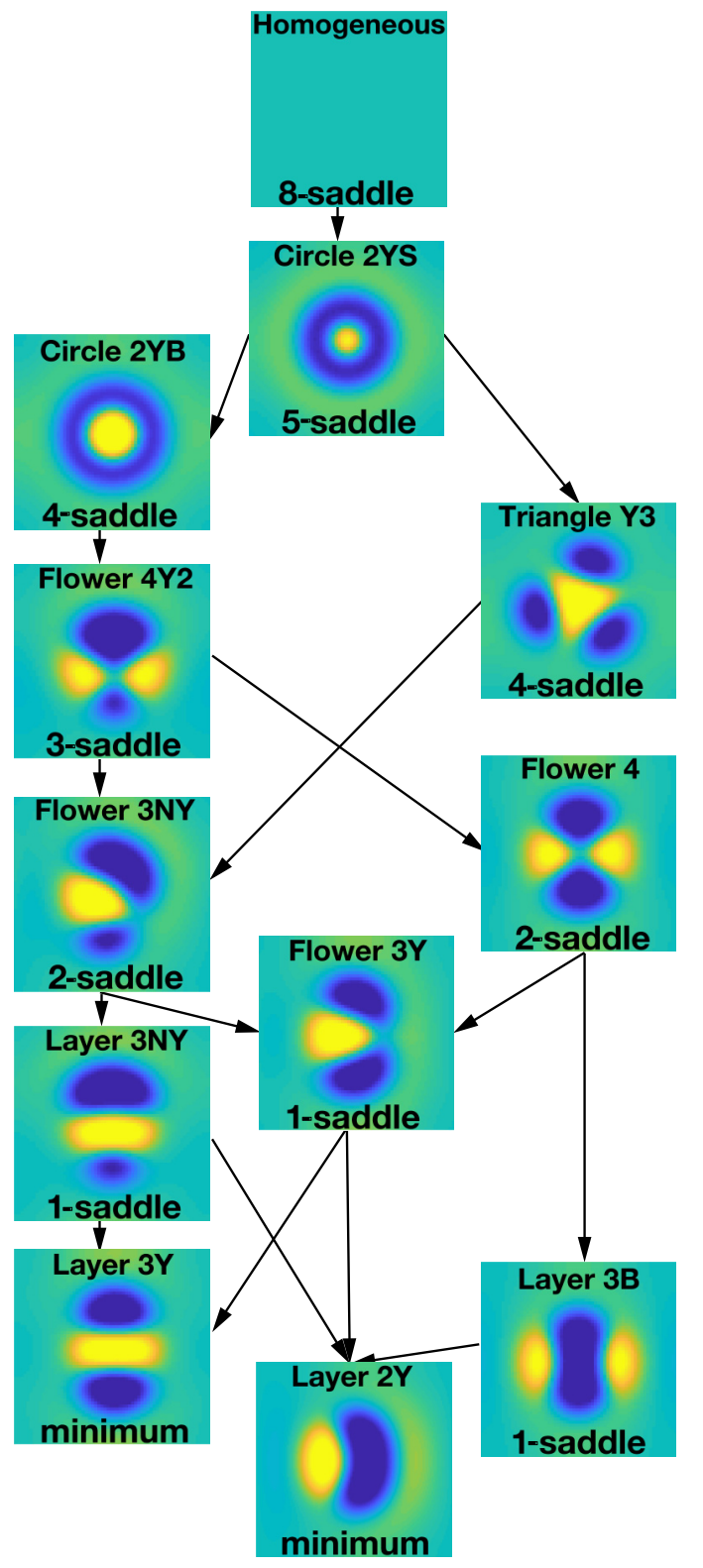

FIG. 4. Solution landscape with selective preference. The height of a phase approximately corresponds to its energy. The $\phi$ plot of each phase is shown in square domain with name on the top and index of corresponding stationary solution on the bottom.

To illustrate the solution landscape more clearly, we classify the stationary solutions from 2-saddles to 6-saddles into four classes: Flower class, Mosaic class, Core-shell class, and Tai-chi class, according to the configuration and connections between them. Mosaic indicates the morphology has multiple alternative yellow and blue blocks. Core-shell represents the class of morphologies that one of the block polymers is surrounded by the other one completely or partially. It is similar to the Circle class, but loses the symmetry of the circle. Tai-chi indicates a class of morphologies that both the blue and yellow parts seem like the yin and yang fishes engaged with each other. If there exists one saddle point in Flower class connecting to another saddle point in Mosaic class, then we present this connection by an arrow from Flower to Mosaic in 


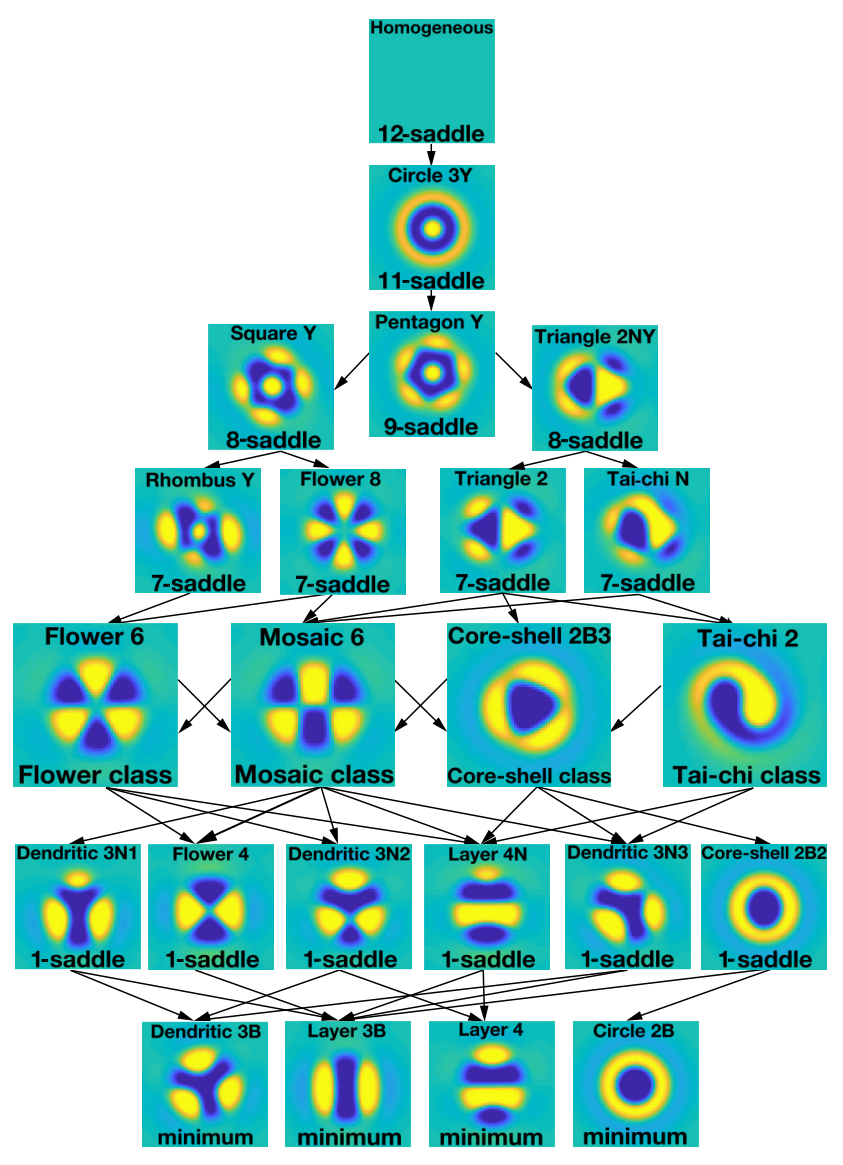

FIG. 5. Solution landscape with equal preference in a larger domain. The stationary solutions with index from 2 to 6 are classified by Flower class, Mosaic class, Core-shell class and Tai-chi class, and the typical solution is inserted in each class as an illustration. The symmetric stationary solutions obtained by switching blue block and yellow one are omitted here.

Fig. 5. Hence the connections between four classes are Flower $\leftrightarrow$ Mosaic $\leftrightarrow$ Core-shell $\leftarrow$ Tai-chi, but the connection from Core-shell class to Tai-chi class has not been found yet. These four classes play key role in connecting the high-index (index $>6$ ) saddle points to the transition states (1-saddles) and minima. For example, we observe the Flower class can be connected from the well defined 7-saddles Rhombus $Y$ and Flower 8 and then connect to the lower-index saddle points Flower 4, Dendritic $3 N 2$ and Layer $4 N$. We can observe the Mosaic class can be connected from almost all 7-saddles and connect to almost all 1-saddles. Here, we treat each class as a whole and omit the detailed connections between the members of each class and the 1 (or 7)-saddles.

Fig. 6 shows four solution landscapes of Flower class, Mosaic class, Core-shell class, and Tai-chi class. In each class, different solutions have subtle differences. The Flower class, including a typical state Flower 6 and many axisymmetric structures, was shown in Fig. 6(a). The Mosaic class with the richest solutions is shown in Fig. 6(b). For instance, 2-saddle Mosaic 6 is the typical mosaic, which looks like a floor tile with three yellow blocks alternating with three blue blocks in the elliptic confinement. The Core-shell class can connect to the circle phase Circle $2 B$ (minimum), shown (a) Flower class

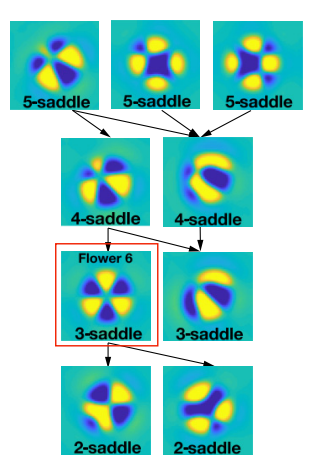

(c) Core-shell class

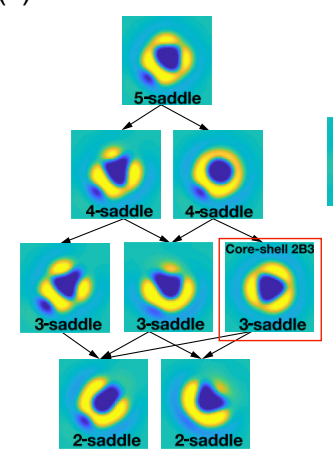

(b) Mosaic class

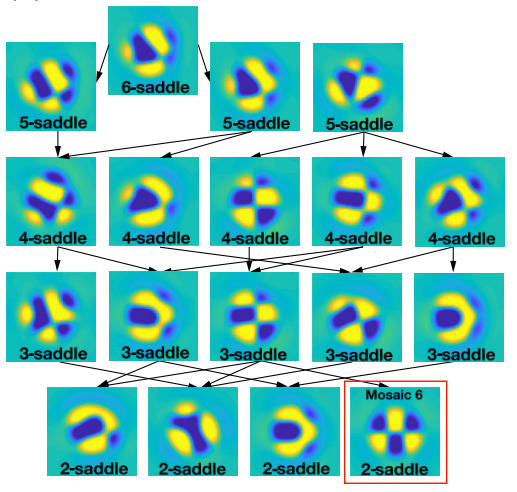

(d) Tai-chi class

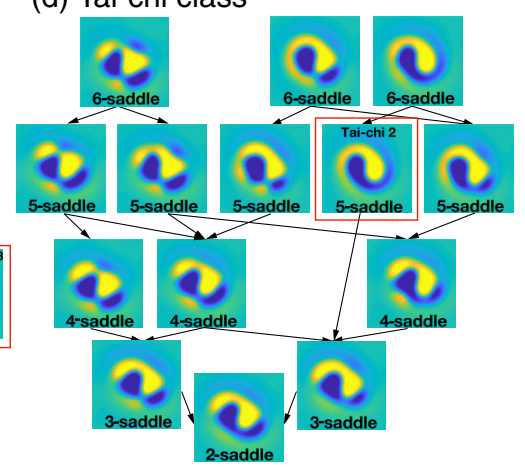

FIG. 6. Solution landscapes of the Flower class (a), Mosaic class (b), Core-shell class (c), and Tai-chi class (d). The typical solutions in Fig. 5: Flower 6, Mosaic 6, Core-shell 2B3 and Tai-chi 2 are marked with red box.

in Fig. 6(c). The typical core-shell is 3-saddle Core-shell $2 B 3$. At last, we also observe the beautiful Tai-chi class in Fig. 6(d). The typical tai-chi phase is 5-saddle Tai-chi 2 with long tails and finally connects to a 2-saddle tai-chi with short tails.

From the solution landscape in Fig. 5, there exist multiple transition pathways between the stable states (Dendritic $3 B$, Layer 3B, Layer 4, and Circle 2B). For example, there are two transition pathways between Dendritic $3 B$ and Layer 3B: Dendritic $3 B \rightarrow$ Dendritic $3 N 1 \rightarrow$ Layer $3 B$ and Dendritic $3 B \rightarrow$ Dendritic $3 N 3 \rightarrow$ Layer $3 B$. However, not all pairs of stable states can be connected by a single transition state. For example, Circle $2 B$ cannot be directly connected to Dendritic $3 B$ or Layer 4 . Thus, the transition pathways between them need multiple transition states. More specifically, the transition pathway between Circle $2 B$ and Dendritic $3 B$ can be Circle $2 B \rightarrow$ Core - shell $2 B 2 \rightarrow$ Lay $3 B \rightarrow$ Dentritic $3 N 3 \rightarrow$ Dendritic $3 B$. Figure 7 shows the 3 -saddle Core-shell $2 B 3$ is the stationary solution in the intersection of the smallest closures of all four stable states. The dynamical pathways from Core-shell $2 B 3$ can be constructed to connect every stable state passing through one 2-saddle and one 1-saddle. Our numerical results highlight the differences between transition pathways mediated by multiple transition states (1-saddles) and dynamical pathways mediated by single high-index saddle point.

\section{Symmetry breaking}

Finally, we investigate the symmetry-breaking phenomena shown in the solution landscapes with different preference 


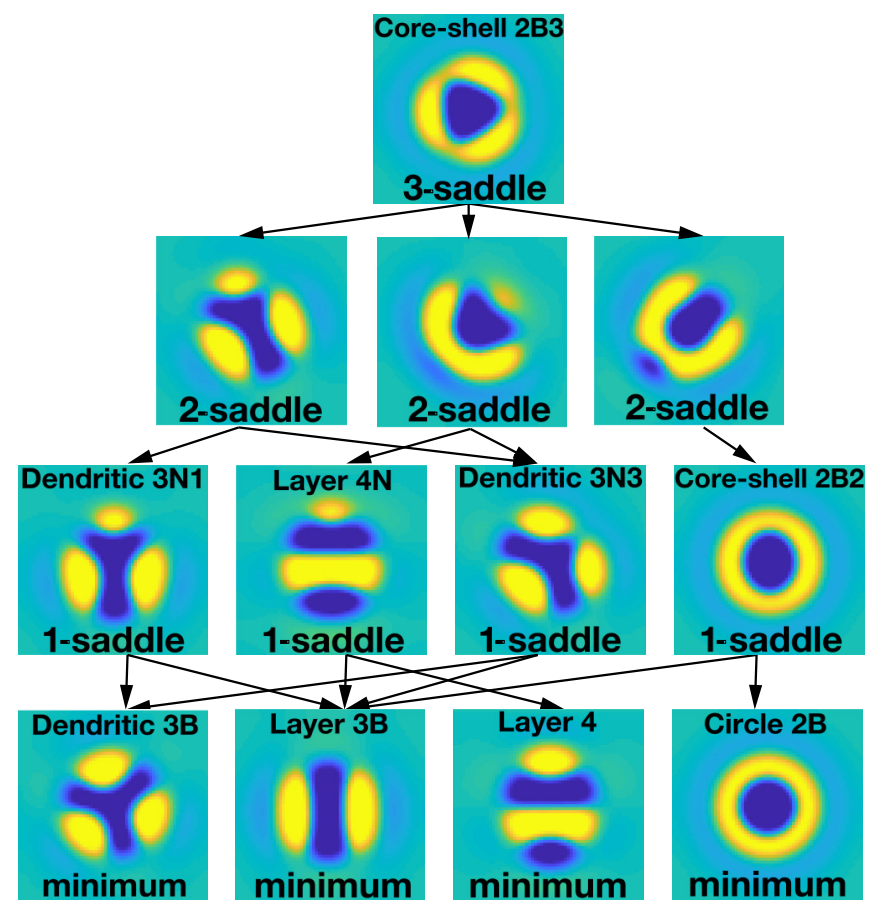

FIG. 7. Solution landscape starting from Core-shell $2 B 3$ solution. All stable states including Dendritic 3B, Layer $3 B$, Layer 4 and Circle $2 B$ are connected by the index- 3 Core-shell $2 B 3$ solution.

intensity and domain size. In view of the previous results, symmetric (reflectional and rotational) properties are quite useful to classify the morphologies for the equal preference as in Figs. 2 and 5. The solutions in $D^{k}$ coincide by itself when rotating by angles $\frac{2 \pi j}{k}, j=1, \ldots, k-1$, or reflecting about the symmetry axes. For instance, the highest-index homogeneous phase has $D^{\infty}$ symmetry (any rotation and reflection is allowed).

In Fig. 8, one can observe the symmetry-breaking process from circular-shape states that has $D^{\infty}$ to the lower symmetric solutions with some $D^{k}(2 \leqslant k<\infty)$ symmetry, e.g., (a) Circle $2 Y S\left(D^{\infty}\right) \Longrightarrow$ Flower 6 and Triangle $Y 3\left(D^{3}\right) \Longrightarrow$ Flower 4 and Layer $3 Y\left(D^{2}\right)$; (b) Circle $2 Y S$ and Circle $2 Y B\left(D^{\infty}\right)$ $\Longrightarrow$ Triangle $Y 3\left(D^{3}\right) \Longrightarrow$ Flower 4 , Layer $3 B$, and Layer $3 Y\left(D^{2}\right)$; and (c) Circle $3 Y\left(D^{\infty}\right) \Longrightarrow$ Pentagon $Y\left(D^{5}\right) \Longrightarrow$ Flower $8\left(D^{4}\right) \Longrightarrow$ Flower 6, Core-shell 2Y3, and Dendritic $3 Y\left(D^{3}\right) \Longrightarrow$ Square $Y$, Rhombus $Y$, Flower 4, Core-shell $2 Y 2$, and Layer $3 Y\left(D^{2}\right)$.

Figure 8 also shows the effect of the preference intensity and the domain size on the symmetric property. In the case of equal preference, there are 8 symmetric solutions in Fig. 8(a). While, as $b_{1}$ is changed from 0 to 0.1 , the selective preference breaks the symmetry and the number of symmetric solutions is reduced to 6 in Fig. 8(b). As stated before, Flower 6 and Triangle B3 disappeared. For the case of equal preference in a larger domain [Fig. 8(c)], the number of symmetric solutions increases to 21 . This is because the larger domain can accommodate more copolymer molecules and multiple finer structures, e.g., the phases Pentagon Y, Flower 8, Square Y, and Rhombus $Y$.

We also note that the highest symmetry $D^{k}$ increases from $D^{3}$ to $D^{5}$ when the domain size $\lambda$ is changed from 1 to (a) equal preference

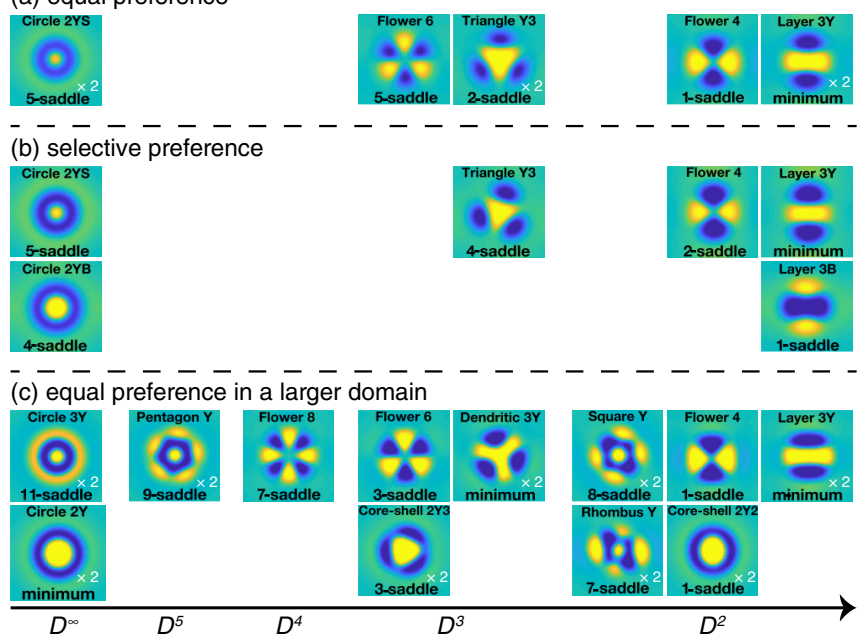

FIG. 8. The comparison of symmetric properties of the solutions in the solution andscapes with (a) equal preference in Fig. 2, (b) selective preference in Fig. 4, and (c) equal preference in a larger domain in Fig. 5. The notation " $\times 2$ " means there exists a pair of phases, e.g., Circle $2 Y S$ and its counterpart Circle $2 B S$.

1.4 , except of $D^{\infty}$. Thus, we expect that, for the stationary solutions of polygonal shapes (except of the homogeneous and circle phases), the upper bound of $k$ in symmetry group $D^{k}$ will continuously increase with the increase of the domain size in the case of equal preference.

\section{v. CONCLUSIONS AND DISCUSSIONS}

In this work, we studied the solution landscapes of the diblock copolymers and homopolymers under 2D confinement with the DCH model. The PSD method is developed to efficiently compute the high-index saddle points with mass conservation and construct the solution landscape of the DCH free-energy functional by coupling with downward and upward search algorithms.

We systematically constructed the solution landscapes by varying the preference intensity and the domain size, which not only provides a global structure of stationary solutions, but also guides our understanding of the dynamical pathways and symmetry-breaking properties of the polymer systems. In the case of equal preference, branching from the homogeneous phase, the solution landscape was obtained, including the Circle-class solutions, Flower-class solutions, Triangleclass solutions and Layer-class solutions. Furthermore, the solution landscape reveals the informative transition pathways between stable and metastable phases. While, with selective preference, the solution landscape loses nearly half of the stationary solutions compared with the one of the equal preference. We can observe the symmetry-breaking phenomenon and the surface has more preference for the blue blocks, which starts to surround the yellow blocks, with the increase of the preference intensity $b_{1}$. These results are consistent to the work by Avalos et al. [21] and the solution landscape provides good guideline for experiments. In the case of equal preference in a larger domain, the solution landscape shows more alternative and interesting stationary solutions. In 
particular, we can classify the solutions as Flower class, Mosaic class, Core-shell class, and Tai-chi class. The relationships between different stable and metstable states are shown by the dynamical pathways connected by a single high-index saddle point (Core-shell 2B3). We further demonstrate that the symmetry-breaking phenomena largely exist in the solution landscapes from high-index saddle points to local minima. The number of stationary solutions with symmetry $D^{k}$ and the upper bound of $k$ increase as the domain size increases.

The numerical results of solution landscapes of the DCH model propose several follow-up questions. First, the extension of the solution landscape from $2 \mathrm{D}$ to $3 \mathrm{D}$ is a fertile ground. The richer and more complex solutions are expected in the solution landscape in the 3D confinement, such as the multipod solution and twisted solution [21]. On the other hand, the viewpoint of symmetry becomes more important in 3D case and more studies will be proceeded in the subsequent work. The numerical difficulty of construction of the solution landscape on 3D comes from the increment of the computational complexity. The degree of freedom $O\left(N^{2}\right)$ for 2D immediately increases to $O\left(N^{3}\right)$ for 3D with $N$ referring to the grid number. Moreover, since the index of the homogeneous state (parent state in the solution landscape) has a large increase from $2 \mathrm{D}$ to $3 \mathrm{D}$, the solution landscapes on $3 \mathrm{D}$ will become more complicated, which brings a huge numerical challenge to finding all solutions.

The framework of solution landscape is believed to be a promising approach to find exotic morphologies with the tight control of the initial conditions (saddle point and associated unstable directions), which overcomes the difficulty of tuning initial guesses to search stationary solutions. This approach can be widely used in the diblock copolymer free-energy functionals. For example, the conjugated diblock copolymers have been used to control over the ratio of two blocks to tune the molecular organization and nanoscale morphology in the solar cells $[60,61]$. We may derive the generalized Landau free-energy model for the diblock-copolymer solar cells and apply the proposed methods to construct the solution landscape of the solar cells systems. To deal with the mass-conservation constraint, the PSD method is developed using the typical $L^{2}$ inner product. We note that the study of SD can also be extended to incorporate the use of different inner products for defining the dynamic systems. One way is to apply the Cahn-Hilliard dynamics using the $H^{-1}$ inner product to avoid imposing the additional conservation constraint [62]. It is interesting to develop the constrained SD method using the $H^{-1}$ inner product to compute anyindex saddle points with mass conservation in the future work.

\section{ACKNOWLEDGMENTS}

This work was supported by the National Natural Science Foundation of China Grants No. 12050002 and No. 11971002. Y.N. gratefully acknowledges the support by JSPS KAKENHI Grant-in-aid No. 20K20341. Y.H. gratefully acknowledges the support from a Royal Society Newton International Fellowship (Grant No. NIF\R1|201143).
[1] F. S. Bates and M. W. Matsen, Macromolecules 29, 1091 (1996).

[2] I. W. Hameley, The Physics of Block Comolymers (Oxford University Press, Oxford, UK, 1998).

[3] F. S. Bates and G. Fredrickson, Phys. Today 52(2), 32 (1999).

[4] R. Deng, S. Liu, J. Li, Y. Liao, J. Tao, and J. Zhu, Adv. Mater 24, 1889 (2012).

[5] A. K. Bhowmick and H. L. Stephens, Handbook of Elastomers (Marcel Dekker, New York, 2001).

[6] G. Holden, Understanding Thermoplastic Elastomers (Hanser Gardner Publications, Munich, 2001).

[7] C. Park, J. Yoon, and E. L. Thomas, Polymer 44, 6725 (2003).

[8] A.-C. Shi and B. Li, Soft Matter 9, 1398 (2013).

[9] X. He, M. Song, H. Liang, and C. Pan, J. Chem. Phys. 114, 10510 (2001).

[10] G. Sevink, A. Zvelindovsky, J. Fraaije, and H. Huinink, J. Chem. Phys. 115, 8226 (2001).

[11] H. Xiang, K. Shin, T. Kim, S. I. Moon, T. J. McCarthy, and T. P. Russell, Macromolecules 37, 5660 (2004).

[12] W. Li, R. A. Wickham, and R. A. Garbary, Macromolecules 39, 806 (2006).

[13] K. Shin, S. Obukhov, J.-T. Chen, J. Huh, Y. Hwang, S. Mok, P. Dobriyal, P. Thiyagarajan, and T. P. Russell, Nat. Mater. 6, 961 (2007).

[14] J. Feng and E. Ruckenstein, J. Chem. Phys. 128, 074903 (2008).

[15] P. Dobriyal, H. Xiang, M. Kazuyuki, J.-T. Chen, H. Jinnai, and T. P. Russell, Macromolecules 42, 9082 (2009).
[16] B. Yu, B. Li, Q. Jin, D. Ding, and A.-C. Shi, Macromolecules 40, 9133 (2007).

[17] R. Yang, B. Li, and A.-C. Shi, Langmuir 28, 1569 (2012).

[18] S. Li, Y. Jiang, and J. Z. Y. Chen, Soft Matter 9, 4843 (2013).

[19] Y. Wu, G. Cheng, K. Katsov, S. W. Sides, J. Wang, J. Tang, G. H. Fredrickson, M. Moskovits, and G. D. Stucky, Nat. Mater. 3, 816 (2004).

[20] S. Thomas, L. Hannah, M. Marzia, V. F.-M. Miriam, R. P. Lorena, and B. Giuseppe, Nano Today 3, 38 (2008).

[21] E. Avalos, T. Higuchi, T. Teramoto, H. Yabu, and Y. Nishiura, Soft Matter 12, 5905 (2016).

[22] E. Avalos, T. Teramoto, H. Komiyama, H. Yabu, and Y. Nishiura, ACS Omega 3, 1304 (2018).

[23] T. Teramoto and Y. Nishiura, JPN. J. Ind. Appl. Math. 27, 175 (2010).

[24] H. Yabu, T. Higuchi, and H. Jinnai, Soft Matter 10, 2919 (2014).

[25] Y. Han, J. Cui, and W. Jiang, Macromolecules 41, 6239 (2008).

[26] M. Pinna, X. Guo, and A. V. Zvelindovsky, Polymer 49, 2797 (2008).

[27] M. Pinna, X. Guo, and A. V. Zvelindovsky, J. Chem. Phys. 131, 214902 (2009).

[28] M. Pinna, S. Hiltl, X. Guo, A. Böker, and A. V. Zvelindovsky, ACS Nano 4, 2845 (2010).

[29] H. Deng, N. Xie, W. Li, F. Qiu, and A.-C. Shi, Macromolecules 48, 4174 (2015). 
[30] P. Chen, H. Liang, and A.-C. Shi, Macromolecules 40, 7329 (2007).

[31] Z. Guo, G. Zhang, F. Qiu, H. Zhang, Y. Yang, and A.-C. Shi, Phys. Rev. Lett. 101, 028301 (2008).

[32] W. Xu, K. Jiang, P. Zhang, and A.-C. Shi, J. Phys. Chem. B 117, 5296 (2013).

[33] T. Ohta and A. Ito, Phys. Rev. E 52, 5250 (1995).

[34] R. Choksi and X. Ren, Physica D 203, 100 (2005).

[35] Y. van Gennip and M. Peletier, Calc. Var. 33, 75 (2008).

[36] K. Glasner, SIAM J. Appl. Math. 79, 28 (2019).

[37] T. Ohta and K. Kawasaki, Macromolecules 19, 2621 (1986).

[38] L. Leibler, Macromolecules 13, 1602 (1980).

[39] Y. Nishiura and I. Ohnishi, Phys. D 84, 31 (1995).

[40] R. Choksi and X. Ren, J. Stat. Phys. 113, 151 (2003).

[41] A. Ito, Phys. Rev. E 58, 6158 (1998).

[42] J. Milnor, Morse Theory (Princeton University Press, Princeton, NJ, 1963).

[43] L. Zhang, W. Ren, A. Samanta, and Q. Du, NPJ Comput. Mater. 2, 16003 (2016).

[44] Y. Li and J. Zhou, SIAM J. Sci. Comput. 23, 840 (2001).

[45] P. Farrell, A. Birkisson, and S. Funke, SIAM J. Sci. Comput. 37, A2026 (2015).

[46] J. Doye and D. Wales, J. Chem. Phys. 116, 3777 (2002).

[47] D. Mehta, Phys. Rev. E 84, 025702(R) (2011).

[48] W. Hao, J. Hauenstein, B. Hu, and A. Sommese, J. Comput. Appl. Math. 258, 181 (2014).
[49] L. Zhang, Q. Du, and Z. Zheng, SIAM J. Sci. Comput. 38, A528 (2016).

[50] J. Yin, L. Zhang, and P. Zhang, SIAM J. Sci. Comput. 41, A3576 (2019).

[51] J. Yin, Y. Wang, J. Z. Y. Chen, P. Zhang, and L. Zhang, Phys. Rev. Lett. 124, 090601 (2020).

[52] J. Y. Yin, B. Yu, and L. Zhang, Sci. China Math. 64, 1801 (2021).

[53] Y. C. Han, J. Y. Yin, P. W. Zhang, A. Majumdar, and L. Zhang, Nonlinearity 34, 2048 (2021).

[54] J. Y. Yin, K. Jiang, A.-C. Shi, P. W. Zhang, and L. Zhang, arXiv:2007.15866 (2021).

[55] Y. Han, Z. Xu, A.-C. Shi, and L. Zhang, Soft Matter 16, 366 (2020).

[56] R. Choksi, M. A. Peletier, and J. F. Williams, SIAM J. Appl. Math. 69, 1712 (2009).

[57] J. Yin, Z. Huang, and L. Zhang, arXiv:2011.13173 (2020).

[58] J. Barzilai and J. M. Borwein, IMA J. Numer. Anal. 8, 141 (1988).

[59] A. Knyazev, SIAM J. Sci. Comput. 23, 517 (2001).

[60] M. He, W. Han, J. Ge, Y. Yang, F. Qiu, and Z. Lin, Energy Environ. Sci. 4, 2894 (2011).

[61] C. Shen, Y.-H. Lee, Y.-P. Lee, C.-J. Chiang, F.-K. Wei, C.-H. Wu, K.-C. Kau, H.-W. Liu, C.-C. Hsieh, L. Wang et al., React. Funct. Polym. 108, 94 (2016).

[62] L. Zhang, J. Zhang, and Q. Du, Commun. Comput. Phys. 16, 781 (2014). 